Stoa

Vol. 3, no. 5, 2012, pp. 87-107

ISSN 2007-1868

\title{
ASPECTOS HERMENÉUTICOS EN LA RECONSTRUCCIÓN DE TEORÍAS
}

\author{
Leticia Minhot \\ Facultad de Psicología- \\ Facultad de Derecho \\ y Ciencias Sociales \\ (Escuela de Trabajo Social) \\ Universidad Nacional de Córdoba \\ leminhot@gmail.com
}

El hombre no encuentra ante sí más que a sí mismo.

Heisenberg

RESUMEN: Moulines, en Pluralidad y recursión, circunscribe la tarea de la filosofía de la ciencia a una empresa reconstructiva del producto de la actividad científica, esto es, las teorías. Para este autor la filosofía, en tanto metateoría, es un discurso que tiene sentido pero que no es ni descriptivo ni normativo sino que su carácter es esencialmente hermenéutico. Lo que intento en este trabajo es proponer una lectura de este carácter hermenéutico que Moulines le atribuye a las metateorías en tanto actividad reconstructiva. Enfocaré mi propuesta en dos aspectos de tal actividad: en la posibilidad o no de un punto de corte en las cadenas interpretativas y en la ausencia del sujeto intérprete.

PALABRAS ClaVE: Reconstrucción de teorías · Interpretación · Hermenéutica · Significado semántico $\cdot$ Significado hermenéutico

ABST RACT: Moulines, in Pluralidad y recursion, restricted the task of the philosophy of science to a reconstruction of the product of scientific activity; that is, theories. For this author philosophy, as metatheory, is a discourse that makes sense, albeit it is not descriptive or normative but is essentially hermeneutic. What I am trying in to do in this work is to propose a reading of this hermeneutic nature attributed to the metatheories as reconstructive activity. I focus my proposal on two aspects of such activity: in the possibility or not of a cut-off point in the interpretative chains and in the absence of the subject interpreter.

KEYWORDS: Reconstruction of theories · interpretation · hermeneutics · semantic meaning $\cdot$ hermeneutical significance 


\section{Introducción}

Moulines, en Pluralidad y recursión, circunscribe la tarea de la filosofía de la ciencia a una empresa reconstructiva del producto de la actividad científica, esto es, las teorías. Esta tarea consiste básicamente en la codificación y sistematización del discurso científico y tiene por principal objetivo la claridad y precisión del mismo mediante un instrumento formal: la teoría de conjuntos. Tal objetivo se subordina a uno cuyas raíces podemos ubicar en Carnap: lograr una fundamentación del conocimiento empírico por medio de ciertos principios de construcción que se aplican a los conceptos envueltos en tal conocimiento. De esta manera la filosofía de la ciencia, concebida como estudio metateórico, permite comprender la estructura semántica de cualquier forma de teorización (Moulines 1991, p. 67). Para Moulines la filosofía, en tanto metateoría, es un discurso que tiene sentido pero que no es ni descriptivo ni normativo, sino que su carácter es esencialmente hermenéutico. De este modo, en ese contexto la tarea de reconstrucción es interpretativa de los aspectos morfológicos de la ciencia, los cuales se revelan mediante formalización y por ello, aquí, interpretar es formalizar. Así, las metateorías de la ciencia son sistemas interpretativos, dando lugar la tarea reconstructiva a una pluralidad de reconstrucciones lógicas de una misma teoría. Cada reconstrucción pone de relieve distintas estructuras de un supuestamente idéntico objeto teórico. Tal suposición es la que me interesa elucidar en este trabajo.

El carácter interpretativo, para Moulines, no es exclusivo de las metateorías, sino que es un rasgo de la misma acción de teorizar. Así, tanto las teorías como las metateorías son sistemas interpretativos. Este carácter es concebido como una invitación a mirar un dominio específico de una manera determinada:

el metateórico, al igual que el pintor, no nos dice: "el mundo es así", sino que nos exhorta "ived el mundo así!" (Moulines 1991, p. 96)

Moulines se diferencia de la propuesta que Sneed ofrece en The Logical Structure of Mathematical Physics (1971), donde prevalece una concepción descriptiva. Para este último, el objetivo de la metateoría es el de clarificar, lo cual se entiende de la siguiente forma:

Tenemos intuiciones razonablemente claras acerca de las pretensiones empíricas de una teoría y de las relaciones lógicas entre ellas. "Razonable- 
mente clara" significa que en la mayoría de los casos podemos pretender confiadamente, por ejemplo, que tal y tal es o no una pretensión de la teoría... "Intuitivo" significa que no apelamos, en realidad, a criterios explícitos que justifiquen tales pretensiones. Con esto, en nuestro punto inicial nos gustaría producir alguna forma comprensiva y perspicaz para demostrar las pretensiones de esta teoría y sus relaciones lógicas. Llamémosle a esto una reconstrucción lógica de la teoría... (Sneed 1971, pp. 3-4)

Se espera que la reconstrucción lógica sea compatible con nuestras ideas intuitivas acerca de la estructura teórica. El problema fundamental con el que se enfrenta esta concepción es la idea de intuición, pues ésta supone la ausencia de pautas explícitas que justifiquen lo que consideramos como las pretensiones empíricas de una teoría dada. Si se admite la posibilidad de que existan diferentes reconstrucciones lógicas de un mismo objeto tenemos dos alternativas ante la pregunta por los criterios con los que evaluamos la compatibilidad entre nuestras intuiciones y la reconstrucción que llevamos a cabo. Un cuerno del dilema sostendría que tal multiplicidad se debe a que existen diferentes intuiciones de las pretensiones de una teoría; el otro cuerno es que se trata de una misma intuición que es susceptible de diferentes descripciones. Si es lo primero, la teoría reconstruida, captada por las diferentes intuiciones que se expresan en cada cuadro descriptivo, no sería la misma pues podrían darse reconstrucciones que le adjudicarían diferentes pretensiones empíricas o diferentes relaciones lógicas entre ellas, y si consideramos a cada reconstrucción como un escorzo de la misma teoría, deberíamos poder decir con base en qué sostenemos el enunciado que afirma tal identidad, pues no hay criterios explícitos que lo justifiquen. En realidad, cada intuición supondría siempre la estructura formal que se utiliza en la descripción que pretende demostrar las pretensiones empíricas de la teoría. Si estamos hablando del segundo, si admitimos que hay una única intuición, recayendo la variedad en las descripciones de la misma, requerimos del postulado de una especie de sujeto epistémico en el cual se unifique tal multiplicidad. Esto último generaría algunas incoherencias con la propia tarea reconstructiva, pues ésta no admite un uso sistemático de un sujeto cognoscente, ya que tal uso implicaría las mismas estructuras formales que se utilizan en la reconstrucción. En tal caso, cada interpretación tendría un sujeto diferente y no podríamos justificar la identidad en- 
tre los mismos, cualquier conceptualización de tal sujeto pertenecería ya a la reconstrucción formal. En síntesis, si varían las intuiciones no puede defenderse la idea de que éstas sean de una misma teoría y si la intuición es una sola, pero lo que varían son sus descripciones, tal pretensión no puede justificarse sin recurrir a las descripciones.

La propuesta de Moulines, que consiste en sustituir descripción por interpretación pareciera que, en principio, evita los cuernos del dilema, ya que cada interpretación es una propuesta de mirar a las teorías de un modo determinado; de modo que no necesitaríamos fundamentar una reconstrucción dada en una intuición previa. Se podría acercar la perspectiva descriptiva a la interpretativa si pensamos que todo cuadro descriptivo contiene, de alguna manera, rasgos interpretativos; sin embargo, habría una diferencia que permanece, aun cuando la descripción se acerque a la interpretación, y que siempre se funda en un punto de corte del círculo interpretativo. Invariablemente hay un punto último, un dado, independiente de la interpretación sobre el cual ésta se justifica. En este caso las intuiciones y los enunciados que describen ese punto de corte, al modo de enunciados protocolares, sería el lugar en donde la cadena de interpretaciones se detiene. Sin este lugar, cada interpretación se justificaría con otras nuevas interpretaciones, con lo que quedaríamos atrapados en un círculo sin justificaciones últimas externas a la actividad hermenéutica. Cuando Balzer y Marcou llevan a cabo la reconstrucción de la teoría temprana del inconsciente de Freud y sostienen la no existencia de criterios independientes y absolutos que permitan decidir la adecuación o no de una reconstrucción, el logro siempre se mide en comparación con otros intentos alternativos (Balzer y Marcou 1989, p. 14). La ausencia de tales criterios podría identificarse con la ausencia de estos puntos que permiten escapar del círculo interpretativo.

Lo que intento en este trabajo es proponer una lectura de este carácter hermenéutico que Moulines le atribuye a las metateorías en tanto actividad reconstructiva. Enfocaré mi propuesta en dos aspectos de tal actividad: en la posibilidad o no de un punto de corte en las cadenas interpretativas y en la ausencia del sujeto intérprete.

El término "hermenéutica" es de vieja data, su sentido original es el de manifestar por medio del lenguaje el logos interior de lo interpretado. La Patrística lo utilizó en el sentido de la manifestación de una 
palabra; esto es, como una traducción de lo oscuro a lo claro. En este contexto, la actitud del intérprete implica una sumisión respetuosa a la intención de la significación encarnada en el texto, monumento u objeto examinado. La hermenéutica, el arte de la interpretación, es suscitada por la existencia de documentos que contienen un sentido, pero lo exhiben de modo confuso y enigmático. Coloco esta referencia histórica no con la intención de hacer una historia del término, sino con la finalidad de elucidar e intentar mostrar las consecuencias epistemológicas y ontológicas que adquieren las diferentes consideraciones de la actividad de interpretar. Una consecuencia ontológica de los sentidos originario y patrístico es la de admitir la existencia de un punto fijo, de un lugar ontológico a partir del cual se podrá captar el mensaje del texto según un esquema de una inteligibilidad unitaria, la idea de una unidad de punto de vista, de una especie de Sinai epistemológico, como la llama Gusdorf, desde el cual se puede asir un determinado objeto. Las distintas interpretaciones del texto suponen un sentido oculto que hay que revelar y cada interpretación es una forma de acercarse cada vez más a ese sentido (Gusdorf 1988, p. 197). Interpretar es, desde esta perspectiva, clarificar, hacer explícito.

Con el romanticismo la hermenéutica opera según otra dimensión cognoscitiva. En el nuevo frente epistemológico que se abre, la idea antes expuesta queda circunscrita a la idea de exégesis como filología bíblica. La hermenéutica, en cambio, es, desde esta dimensión, la comprensión, desde una conciencia actual, de un objeto cultural situado en un horizonte diferente. La comprensión será la restauración completa del horizonte cultural del pasado lejano basándose en el supuesto de una transformación permanente que le permite pasar al intérprete de su universo de origen al universo del texto que quiere interpretar. La hermenéutica se hace historia, o mejor aún, arqueología (Gusdorf 1988, pp. 201-202).

Sin embargo, hay en los dos sentidos algo en común, algo fijo que debe ser revelado; esto es, el sentido que reside en el texto a interpretar. La historicidad del segundo significado de "hermenéutica", hace historia del punto fijo del sentido anterior, pero los textos tuvieron un significado para sus autores o contemporáneos y es lo que se busca restaurar. Si bien se restaura situándolo en su tiempo y con la conciencia de la distancia con respecto al intérprete, continúa habiendo sumi- 
sión ante el texto. El sentido continúa siendo algo que se esconde, que hay que develar. Por un lado, cuando lo oculto y enigmático es propio del texto, la actividad interpretativa consiste en descifrar: es exégesis. Por otro lado, cuando lo oculto y enigmático se debe a la distancia, la interpretación se vuelve arqueología: restaura completando las partes dañadas por el tiempo. En ambos casos hay un dado que funda y justifica la interpretación y, por ende, sirve de pauta absoluta y única para evaluar a ésta. El problema que aquí se presenta consiste en cómo reconocemos tal dado para que nos sirva de criterio.

La discusión epistemológica en torno a esta problemática es heredera directa de las discusiones teológicas del siglo xvi. En las primeras protestas en contra de los principios católicos, Lutero argumentó que las proposiciones religiosas no debían ser juzgadas según su acuerdo con la tradición de la iglesia católica expuesta en concilios y decretos papales, proponiendo un nuevo criterio del conocimiento religioso. Éste último se asentó en la idea de que la conciencia es compelida a creer que las escrituras son verdaderas. Por siglos se consideró que sólo las proposiciones autorizadas por la iglesia podrían establecer una verdad religiosa. Como sostiene Popkin "la caja de Pandora que Lutero abrió en Leipzig tuvo consecuencias que llegarían muy lejos, no sólo en teología, sino también a través de todo el campo intelectual de Occidente" (Popkin 2003, p. 5).

¿Cómo defender una pauta de verdad? No podemos apelar a una evidencia sin caer en una petición de principio. En términos de Popkin, el valor de la evidencia depende del criterio y no viceversa; sólo otros criterios justifican un criterio. En síntesis, la disputa se centró alrededor de estos dos argumentos: los católicos fundaron su pauta en la tradición, los protestantes en la iluminación. Los reformadores, por un lado, defendían un criterio individual y subjetivo el cual era, a su vez, utilizado como medida objetiva para condenar las herejías; por otro lado, los católicos consideraban que este criterio llevaría a la anarquía religiosa y sostenían que cualquiera podría apelar a su propia conciencia diciendo que lo que era verdad para él era la verdad. Mientras para Lutero la conciencia es compelida, para Erasmo las Escrituras no son tan claras y hay lugares que resultan oscuros para la comprensión del ser humano. Ante la dificultad que reside en establecer el verdadero significado de las Escrituras, ¿̇por qué no aceptar la solución tradicio- 
nal ofrecida por la iglesia? La actitud prudente de Erasmo consistía en que él no se consideraba a sí mismo capaz de distinguir la verdad de la falsedad con certeza, por lo que él depositaba toda la responsabilidad en la institución que durante siglos había establecido tal distinción. Lutero insistía en la certeza subjetiva, en la compulsión de la propia conciencia a aceptar la verdad, en contra del canon que toma a la tradición como autoridad. Sin embargo, este criterio esta sometido a serias objeciones: si la conciencia de todos los hombres es compelida por la misma verdad ¿̇con base en qué se justifica la condena de las herejías?, las cuales son el resultado de diferentes interpretaciones de un mismo texto y ¿cómo se comprende el caso Servet si se sostiene, con Calvino, la norma de la persuasión interna? El problema al que intentan responder estos argumentos es el del criterio del conocimiento y esta crisis que representó la Reforma se extendió desde la teología a la filosofía, siendo crucial en la emergencia de la filosofía moderna. Partir de que hay algo fijo que debe ser clarificado mediante la interpretación conlleva siempre el dilema que enfrentaron los teólogos: ¿quién es más adecuado para captar eso dado, la tradición de la institución que fuera o las intuiciones de cada intérprete? Trasladando esta cuestión a la problemática que en este trabajo analizo podríamos reformularla de la siguiente manera: ¿quién es más adecuado para captar ese dado, la tradición de la comunidad científica o las intuiciones de cada intérprete? Lo primero supone apelar a un criterio pragmático y lo segundo supondría apelar a un nuevo criterio que justifique nuestras "intuiciones razonablemente claras" en las cuales confiamos. El primer criterio se presenta en principio como más saludable, el problema que se presenta es que no tenemos una única tradición en la comunidad científica que se presente a lo largo de la historia de modo continuo y constante como el caso de la tradición católica y rara vez tenemos un único texto en el que las pretensiones empíricas de una teoría aparezcan de una vez. ¿A la tradición de qué comunidad debemos apelar? ¿A la de la comunidad en la que el autor se ubicó o a la actual? Apelar a la comunidad contemporánea a la emergencia de la teoría supone un nuevo trabajo interpretativo, el cual se justificará con nuevas interpretaciones.

Propongo tomar el camino desde otro punto, el cual nos permita evitar lo dado como punto de corte de nuestras interpretaciones. En 
cualquier sentido de hermenéutica, ésta trata con los significados; si bien aquí lo que se interpreta son estructuras que se revelan en la formalización, tales estructuras son formas semánticas. Justamente, lo que intento mostrar es que no podemos separar en la actividad de reconstruir teorías la forma del contenido, pues los contenidos y las formas de la ciencia son inseparables. Entonces analicemos un poco el concepto hermenéutico de significado.

Para Taylor este concepto aplicado a textos, monumentos, acciones, situaciones, es diferente del concepto lingüístico de significado. El significado hermenéutico es el concepto de algo para un sujeto determinado o para una comunidad y existe en un campo, eso es, con relación a la significación de otras cosas. El significado lingüístico es también un concepto de algo para un sujeto y existe en un campo de significados, pero adicionalmente liga un significante y un referente. Moulines define el atributo "semántico" de la siguiente forma:

será considerado semántico cualquier enfoque filosófico que arranque de la reflexión sistemática sobre el supuesto básico de que el lenguaje tiene significado, o dicho más exactamente, de que ciertos elementos lingüísticos se hallan en una relación constitutiva con algo extralingüístico, relación imprescindible para poder ser considerados justamente parte del lenguaje (Moulines 1991, p. 130).

Taylor, al sostener que un sentido es siempre de algo, se compromete con la posibilidad de distinguir entre un elemento dado -situación, acción, enunciación, etc.- y su significado, aunque no en el sentido de que estén físicamente separados. En otras palabras, no hay sentido sin un sustrato. Podemos, sin embargo, separar dos descripciones, una caracterizada en términos de sus conceptos para un sujeto y otra libre de significado. Podemos separar entre la acción "Bruto mató a César" y su significado en la historia de Roma (Taylor 2005, p. 152). Este es el punto donde difieren McGuire y Tuchanska (2000), pues para ellos no hay sustrato, no hay algo que deba ser estudiado previamente a los sentidos pues es a través del complejo de significados que algo viene a nosotros, siendo imposible la segunda descripción. La idea de significado hermenéutico que aventuro aquí se caracteriza por los dos siguientes rasgos: el primero, siguiendo a Taylor, conserva lo que lo diferencia con el lingüístico o semántico; esto es, carece de referente; el segundo, siguiendo a McGuire y Tuchanska, señala que, si bien todo 
significado es el concepto de algo, ese algo no es previo al sentido sino que es constituido por esta significación. No se trata de interpretaciones diferentes de un sustrato, pues éste no es separable del acto de interpretar. El significado de un texto no es ni una esencia que se revela en la especulación filosófica ni ésta tiene la tarea de reconstruir el sentido que el texto tuvo para sus autores o contemporáneos. En oposición a las concepciones exegéticas y arqueológicas de la hermenéutica, llamaré a la que aquí propongo "constitutiva", pues la interpretación así concebida no busca clarificar algo dado, sino que instituye significados. De este modo, el significado semántico está contenido en el significado hermenéutico. Por ende, una caracterización de toda forma de teorización como interpretativa implica que la comprensión de la estructura semántica de tales formas supone siempre una hermenéutica constitutiva. Bajo esta hipótesis intento ofrecer una relectura de la exhortación "iVed el mundo así!".

Moulines sostiene que:

La interpretación o reconstrucción de la morfología de la ciencia que se intenta en nuestra disciplina es, en lo fundamental, de un tipo muy parecido a las que realizan, con sus respectivos "materiales", diversas formas de arte, en especial las artes plásticas y las novelas. El conocimiento que proporcionan la filosofía de la ciencia sobre su objeto (a saber, las formas de la ciencia) es, en consecuencia, un conocimiento de orden estético y su legitimidad, la misma que la que poseen otras actividades artísticas (1991, p. 89).

Una teoría, tanto científica como metacientífica, es para Moulines una visión de las cosas o una propuesta para mirar de determinado modo. De manera análoga, para Eliot el arte tiene como función imponer un orden creíble a la realidad ordinaria para de allí obtener alguna percepción de un orden real (1951, p. 43). Una reconstrucción de una teoría científica impone a teorías familiares un nuevo orden, el cual es como una nueva luz que nos permite ver nuevas formas. Los componentes estéticos dependen de si se trata de arte, de ciencia o metaciencia. En el caso de la interpretación filosófica de la ciencia estos componentes están íntimamente relacionados con el carácter reconstructivo de la filosofía, pero la idea de reconstrucción no debe confundirse con la de restauración. El metateórico filosófico es como un pintor, no como un restaurador (aunque a veces haya pintores que 
cumplan el oficio de restauradores de cuadros de otros pintores y aunque a veces haya filósofos historiadores que crean que restauran). Una reconstrucción no busca restaurar las partes dañadas por el tiempo ni reconstruir el sentido - ni las estructuras profundas- que la teoría tuvo para su autor. La reconstrucción no es una arqueología. Sin embargo, hay pasajes en donde Moulines parece aproximar la empresa reconstructiva a la exegética. Consideremos el siguiente fragmento del texto mencionado:

Reconstrucciones no-equivalentes de la misma teoría científica pueden ser igualmente valiosas en el sentido de que ponen de relieve distintas estructuras profundas, todas igualmente interesantes, del mismo objeto teórico (Moulines 1991, p. 95).

La pregunta que queda aquí sin respuesta es: ¿̇cómo sabemos que se trata del mismo objeto teórico? La expresión "mismo objeto teórico" hace pensar que éste es el sustrato del cual la actividad metateórica es su interpretación, en cuyo caso la interpretación no es constitutiva. Moulines analiza la situación apelando a un ejemplo pictórico. En tal ejemplo se afirma que Cristo crucificado es el mismo objeto simbólico interpretado de modos distintos por Velásquez y Dalí. Ahora bien, ¿en base a qué criterios afirmamos la identidad de lo representado? El Cristo de Dalí es un Cristo visto desde arriba y este cambio de perspectiva supone todo un complejo de significados que traen algo muy distinto a lo que trae el cuadro de Velásquez. Las estructuras profundas de ambos cuadros son distintas, nadie ignora el rol de la mirada en la representación del sentimiento religioso y los distintos escorzos no son meras miradas desde lugares diferentes de lo mismo. En realidad, la perspectiva en sí contiene ya una interpretación, por lo que su presencia o ausencia supone una decisión interpretativa previa. Panofsky considera la perspectiva como una forma simbólica, entendida ésta al modo de Cassirer, en el sentido de que un determinado contenido se une a un signo sensible concreto y se identifica con él (Panofsky 1927, p. 24). Resulta muy difícil separar forma de contenido. La pregunta que deseo plantear es la referida a la identidad de objetos que vienen en redes de significados diferentes, en órdenes formales diferentes. Si la actividad reconstructiva trabaja revelando estructuras profundas, ¿̇con base en qué criterios podemos establecer la identidad entre objetos teóricos de los que se develan estructuras profundas di- 
ferentes? Si las mismas sólo son reveladas por sistemas interpretativos se requeriría de un criterio externo a tales sistemas para afirmar que objetos que pertenecen a sistemas diferentes son el mismo, pero tales criterios externos pertenecen a su vez a nuevos sistemas interpretativos. Podríamos utilizar un criterio a lo Kripke y decir que el nombre "Cristo crucificado" es una especie de designador rígido que nombra a lo que está pintado como un referente único, por lo que el enunciado que afirma la identidad de ambos objetos es un enunciado que expresa una verdad necesaria. En ese caso, los Cristos de Velásquez y de Dalí serían como mundos posibles y el nombre "Cristo crucificado" designaría al mismo objeto, pero ¿bajo qué criterios legitimamos como necesaria la verdad que afirma la identidad entre ambos Cristos? Coincido plenamente con Moulines en el sinsentido de la expresión "El Cristo de Velásquez es más verdadero que el Cristo de Dalí" y la expresión no tiene sentido porque no hay un Cristo objeto simbólico como límite asintótico de las representaciones de Velásquez y de Dalí. Ni Velásquez ni Dalí hacen exégesis o historia; hacen ontología, instituyen un significado, un símbolo. Del mismo modo, lo que defiendo aquí es el carácter constitutivo de la reconstrucción de teorías, por lo que no podemos establecer la identidad de objetos teóricos revelados por reconstrucciones no equivalentes. La idea de exégesis requiere que el objeto teórico sea el mismo, pues es el punto fijo al cual se acercan las diferentes interpretaciones. Si los aspectos morfológicos son aspectos esenciales y cada formalización revela aspectos diferentes, entonces el enunciado que afirma la identidad del objeto teórico ¿̇a qué sistema interpretativo pertenece? Si los aspectos morfológicos que se ponen de relieve en una formalización son escorzos de un objeto teórico no tenemos formas de saber si en formalizaciones diferentes se trata del mismo objeto, pues cada formalización sólo deja ver ese escorzo. Ninguna interpretación puede darnos algo más allá que la perspectiva que deja ver; una reconstrucción formal no puede ser exégesis. En realidad, la idea de exégesis nos compromete con una entidad trascendente al sistema interpretativo y en ausencia de ésta resulta difícil justificar el modo exacto en que distintos sujetos -interpretaciones diferentestratan con el mismo objeto, para el caso, el mismo objeto teórico.

Una interpretación es constitutiva en el sentido de que lo interpretado depende solamente de las propiedades formales del sistema in- 
terpretativo, de modo que el objeto en sí no está ya hecho, sino que es el resultado de un modo de tomar el mundo, es el resultado de aquella exhortación "iVed el mundo así!". En ese modo de tomar el mundo entran desde los aspectos formales del sistema interpretativo al establecimiento de lo que previamente tiene una dirección abierta a posibilidades. El sentido semántico de significado es denotativo y requiere del objeto referente, pero el sentido hermenéutico implica organización, configuración y prescinde de tal referente. El objeto interpretado y sus aspectos dependen de la organización, la cual forma, relaciona y distingue a los objetos. Es así que la exhortación es constitutiva y el énfasis recae en el sistema interpretativo y no en lo interpretado.

Para Goodman "el hombre del Paisaje con Cazador de Rembrandt no es probablemente una persona real, es justamente el hombre del grabado de Rembrandt. En otras palabras, el grabado no representa a un hombre, sino que es simplemente un cuadro-de-hombre, y más concretamente el hombre-del-cuadro-de-Rembrandt-Paisaje-con-Cazador y aunque el hombre allí pintado fuese un hombre real, su identidad importa tan poco como el grupo sanguíneo del artista" (1968, pp. 42).

Los Cristos de Velásquez y de Dalí no denotan al Cristo histórico como mismo objeto simbólico; hablar del Cristo de Dalí es hablar del Cristo-del-cuadro-de-Dalí, hay un nuevo objeto simbólico.

Si admitimos, con Moulines, que en la reconstrucción se interpreta formalizando, ¿̇cuál es el alcance epistemológico y ontológico de este supuesto? Ante diferentes formalizaciones de una misma teoría, ¿̇en qué fundamos el criterio de identidad, que no sea la sola declaración de la intencionalidad del intérprete, que nos permite hablar del mismo objeto teórico? En An Arquitectonic for Science:

Primero, desarrollamos ciertas herramientas metodológicas formales y entonces usamos las herramientas para representar la estructura de algunos ejemplos específicos de ciencia empírica y su desarrollo en el tiempo. En términos que se dan ahora entre trabajadores en inteligencia artificial, desarrollamos un "esquema de representación” para el conocimiento científico. Probamos la adecuación de ese esquema al aplicarlo en la reconstrucción de ciertos fragmentos de la ciencia empírica. El criterio primario por el cual juzgamos la adecuación de nuestra reconstrucción es éste. La reconstrucción de cada pieza particular de la ciencia debiera "generar" de un modo natural, estandarizado y sistemático las propiedades esenciales de esa pieza y las pretensiones centrales asociadas a ésta por los usuarios ha- 
bituales de esa pieza (los expertos) (Balzer, Moulines y Sneed 1987, pp. xvii-xviii). ${ }^{1}$

Un esquema de representación es un sistema interpretativo y no descriptivo, por lo que las propiedades esenciales que se generan no son las de la pieza tomada en sí misma, independientemente del sistema utilizado. El fragmento interpretado siempre se nos presenta en las redes de un esquema de representación. ¿Cuál es el criterio para evaluar la adecuación? El problema, como ya señalamos, es nuestro acceso a esas propiedades esenciales. Con Sneed en An Arquitectonic for Science, a través de nuestras intuiciones, el criterio reside en la tradición a la que pertenecen los expertos; y con Balzer y Marcou no existen pautas independientes y absolutas que permitan decidir la adecuación o no de una reconstrucción. En realidad, ninguna perspectiva ofrece un criterio absoluto o independiente de fidelidad con el objeto representado.

Goodman (1968) distingue dos modos de uso del término "representar" que podemos aplicar aquí al esquema de representación construido con ciertas herramientas metodológicas formales. Uno de esos sentidos es el denotativo, el cual coloca el énfasis en el objeto representado; esto es, el objeto al cual se refiere la representación. Así, un cuadro tiene que denotar a un hombre para representarlo. La representación denotativa es una relación diádica entre un cuadro y aquello a lo que el cuadro se refiere, pero no siempre utilizamos el término "representación" en el sentido denotativo; a veces el término se usa no para hablar del objeto representado externo al cuadro, sino para decir qué clase de cuadro es; la utilizamos como descripción del cuadro y, en este último sentido, la representación es monádica. Goodman utiliza la expresión cuadro-de-hombre para abreviar "cuadro que representa a un hombre"; en este sentido un cuadro puede no representar nada. Es necesario que un cuadro denote un objeto $x$ si pretende representarlo, pero para que el cuadro sea una representación-de- $x$ no requiere representar a $x$ (1968, p. 42). Un aspecto no es el objeto desde una cierta distancia, un cierto ángulo y bajo una cierta luz, sino que es el objeto tal como lo contemplamos, al representar un objeto lo consumamos (Goodman, 1968, pp. 26 y ss.), los objetos son inmanentes a

${ }^{1}$ El énfasis es mío 
las representaciones. En este sentido, decir que la reconstrucción genera los aspectos esenciales no significa que estos son independientes y previos a la tarea de reconstruir, son aspectos esenciales relativos al instrumento formal que se ha desarrollado previamente. Tal como explicita Goodman, la elección y el tratamiento del instrumento participan en la interpretación (1968, p. 27, n.8). El significado es una condición formal de la inteligibilidad de las teorías, una configuración de las mismas. Como aquí la interpretación que lo pone en juego es una formalización, éste no es un carácter sustantivo de las teorías sino la condición del instrumento que se utiliza en la formalización.

El esquema representativo se apodera performativamente, es una ejecución que se apropia del modo de ser del objeto. La representación en el sentido monádico que señalara Goodman consuma una configuración, una ordenación.

Balzer, en Teorías empiricas: modelos, estructuras y ejemplos, sostiene que:

La meta de nuestra exposición no es introducir al lector para que prosiga la investigación especializada en el sector correspondiente; la meta consiste en presentarle del modo más preciso posible la estructura conceptual y lógica de la teoría de la que se trate. [... ] En primer lugar, la reelaboración y penetración epistemológicas de las teorías empíricas sirve para clarificarlas, para precisarlas...(Balzer 1997, p. 18).

Si pretendemos que la reconstrucción formal metateórica sea una interpretación y no una descripción, la meta de claridad y precisión debe ser leída de otra forma. Esta idea, que se adquiere en el proceso de reconstrucción, parece llevarnos al supuesto de que hay un sentido, si no oculto y enigmático, al menos confuso que pone en marcha el proceso en cuestión del mismo modo que el sentido no manifiesto de los textos sagrados puso en marcha la hermenéutica como exégesis. De esta forma la formalización es una especie de cábala epistemológica que devela un sentido confuso. ¿Cómo entender el ideal de claridad desde una perspectiva hermenéutica constitutiva del significado?

¿Son las formalizaciones una hermenéutica en sentido constitutivo? Esto es, ¿̇generan las formalizaciones los objetos que interpretan? Si tomamos por un momento la idea de que la interpretación es de algo dado previo, tendríamos entonces un proceso similar al de una epojé en el que se suspende todo juicio interpretativo y nos enfrenta- 
mos directamente con los aspectos morfológicos de una teoría, pero es insostenible hablar de aspectos morfológicos al modo de un dado no conceptualizado, pues aquí ya estamos suponiendo una interpretación. Esta suspensión del juicio interpretativo haría ciega la intuición de tales aspectos. Las estructuras profundas de una teoría, sus propiedades esenciales, se develan bajo un esquema de representación; esto es, en una formalización que interpreta. Entonces tal intuición no nos permite una comprensión de esas propiedades esenciales, pero ¿̇bajo qué supuesto podemos afirmar que existe una intuición inocente de toda interpretación? Si comprender es captar estructuras y comprendemos interpretando, esto es, formalizando, ċqué es lo que se capta en una intuición ciega? ¿Y de qué modo puede servirnos para comparar interpretaciones diferentes?

El punto es que, dado que comprendemos formalizando cuando desde una comprensión formal vamos a interpretarlas, lo que descubrimos es el esquema representacional en la que estamos. Lo que se nos devela es el sistema formal desde el cual interpretamos; esto es, Richard Montague descubre estructuras de lógica de primer orden, $\mathrm{Pa}$ trick Suppes conjuntos, y si se reconstruye la misma teoría utilizando lógica de categorías, se descubrirán categorías. Siempre estamos confinados al sistema que empleamos cuando nos referimos a aquello que reconstruimos, por lo que nuestros objetos teóricos y sus propiedades esenciales son la consecuencia del sistema formal que utilizamos. Por lo anterior, carece de sentido hablar del mismo objeto teórico independientemente del sistema que se elige para su reconstrucción. Pero tampoco tiene sentido afirmar que son objetos teóricos diferentes, porque el mismo problema se presenta aquí, ¿̇esde dónde afirmamos sus diferencias? La formalización de una teoría no es un acto referencial, es un rehacer, y los aspectos morfológicos que revelan no son previamente intuidos, sino que son producidos, se descubre lo que se diseña o como sostiene Goodman, comprensión y creación van de la mano (1978, p. 43). Esto sería algo similar a lo que Heisenberg sostiene con respecto a los componentes mínimos de la materia:

Cuando se trata de los componentes mínimos de la materia, en cambio, aquel proceso de observación representa un trastorno considerable, hasta el punto de que no puede ya hablarse del comportamiento de la partícula prescindiendo del proceso de observación. Resulta de ello, en definiti- 
va, que las leyes naturales que se formulan matemáticamente en la teoría cuántica no se refieren ya a las partículas elementales en sí, sino a nuestro conocimiento de dichas partículas [...] La noción de realidad objetiva de las partículas elementales se ha disuelto por consiguiente en forma muy significativa, y no en la niebla de alguna noción nueva de la realidad oscura o todavía no comprendida, sino en la transparente claridad de una matemática que describe no el comportamiento de las partículas elementales, pero sí nuestro conocimiento de dicho comportamiento. (Heisenberg 1955 , p. 14).

Una interpretación formal en sentido constitutivo es una comprensión de formas y estructuras, pero tal comprensión es constitutiva, es una forma de realización. Comprender no es interpretar algo, sino que al interpretar a través de un sistema formal algo emerge y esto no es un sustrato independiente de la interpretación. Como aquí se interpreta formalizando lo que se realiza, lo que emerge son los aspectos morfológicos de las teorías, los cuales dependen del aparato formal que se utiliza para la reconstrucción. Estos aspectos vienen a nosotros a través de una determinada herramienta formal, por ello no tiene sentido decir que una axiomatización es más verdadera que otra ni tenemos criterios independientes para evaluar su adecuación. El texto en el que aparece la teoría interpretada no puede ser pensado como el punto fijo que esconde el sentido último hacia el cual se acercan las diferentes interpretaciones formales: comprender es descubrir la interpretación formal desde la cual reconstruimos. Los aspectos morfológicos son estructuras de nuestro aparato formal, son las propiedades de la herramienta formal; interpretar es una forma de hacer objetos y, si se interpreta formalizando, lo que se hace, lo que se construye, son estructuras formales. Esto significa que esas estructuras, que esos aspectos morfológicos, no están en las teorías sino en el aparato formal que las interpreta, son el resultado de ese rehacer. No se trata ni de una descripción ni de una exégesis, sino de una interpretación de nuestras propias herramientas metateóricas, las cuales nos gobiernan en todo proceso reconstructivo. Lo que interpretamos es el modo de gobierno del esquema de representación utilizado. Desde esta perspectiva, el ideal de claridad debe entenderse como la explicitación de los aspectos formales implicados por el instrumento interpretativo. No debemos pensar que esto nos lleva -usando una expresión de Gusdorf- al ateísmo epistemológico: el relativismo, pues no hay una renuncia a una 
claridad objetiva. Lo que se hace claro, en definitiva, son las condiciones de interpretación. Parafraseando a Heisenberg, para disolver las oscuridades se debieron hallar fórmulas que expresen, no a las teorías científicas, sino el conocimiento que de ellas tenemos, renunciando a un modo de concebir una descripción de ellas: "La incidencia del método modifica su objeto y lo transforma hasta el punto de que el método no puede distinguirse del objeto" (1955, p. 27).

Una interpretación es una perspectiva, un mirar "a través de", tal como definiera Durero a esta última. En tanto perspectiva, la interpretación puede ser analizada de dos modos diferentes. Por un lado coloca la apariencia subjetiva del intérprete al introducir un momento individual y contingente; por otro, es un instrumento que garantiza racionalidad y objetividad, ambos modos convergen si llevamos la analogía con la perspectiva pictórica hasta sus últimas consecuencias. La concepción perspectivista, nos indica Panofski (1927, p. 53), se funda en la voluntad de crear el espacio figurativo. En esta concepción, la especialidad obtiene del sujeto las propiedades que la caracterizan pero a la vez la liberan de todo subjetivismo. La idea de interpretación como perspectiva impide dos accesos: a la cosa en sí -a propiedades esenciales de lo interpretado- y al dominio de lo psicológico del intérprete. Las leyes formales del sistema interpretativo son el "a través de" lo que se ve y desplazan el acto visionario de la vivencia inmediata del observador. El momento individual y contingente no es la creación psicológica de un espacio visual propio, es más bien un momento objetivo donde el centro visual no es un centro psicofisiológico, sino un espacio matemático fijo, un único ojo inmóvil que genera una discrepancia entre lo que las cosas son y la construcción que hace posible los objetos. La perspectiva, como la interpretación, introduce la distancia entre el "en sí" y el esquema de representación. Distancia que en la exégesis se debió a lo esquivo del texto, en la arqueología a la dimensión temporal, y ahora al carácter constitutivo del sistema interpretativo.

Panofsky, al referirse al espacio de la perspectiva pictórica, sostiene que si se quiere garantizar la construcción de un espacio totalmente racional éste tiene que ser infinito, constante y homogéneo, lo cual es garantizado por la "perspectiva central" del ojo único y fijo (1927, pp. 
12 y ss.). Algo análogo a tal ojo es el observador ideal que construye conceptos tal como lo concibiera Carnap en el Aufbau:

Estos presupuestos implican verdaderamente una audaz abstracción de la realidad (si por 'realidad' entendemos en este caso la efectiva impresión visual en el sujeto). La estructura de un espacio infinito, constante y homogéneo (es decir un espacio matemático puro) es totalmente opuesta a la del espacio psicofisiológico. La construcción perspectiva exacta abstrae de la construcción psicofisiológica del espacio, fundamentalmente: el que no sólo es su resultado sino verdaderamente su finalidad, realizar en su misma representación aquella homogeneidad e infinitud que la vivencia inmediata del espacio desconoce, transformando el espacio psicofisiológico en espacio matemático. (Panofsky 1927, pp. 13-14)

"iVed el mundo así!" es la exhortación de toda actividad teorizadora que propone un orden para ver "a través de". "iVed el mundo así!" es el postulado de un ojo fijo que identifica un contenido con un modo de ver. Ojo fijo que funciona como constructor sistemático de objetos. La meta de la claridad, leída desde esta concepción perspectivista de la interpretación no supone claridad o hacer explícitas propiedades esenciales que las teorías nos ocultan, claridad significa aquí la transposición de la objetividad interpretativa a la objetividad del espacio de un esquema formal de representación; esto es, la interpretación es objetiva porque se da en un determinado espacio formal que nos permite recorrer todos sus puntos paso a paso, pues su homogeneidad se funda en el conjunto de sus funciones lógicas y matemáticas. Éstas son las que garantizan que puedan construirse objetos idénticos, lo que significa que encajan en el mismo esquema de representación, en diferentes lugares del espacio. Pero la identidad que aquí se garantiza es la que se da entre objetos del mismo espacio: identidad de formas de diferentes teorías. Lo que no podemos garantizar es la identidad de objetos que pertenecen a espacios diferentes, pues la modificación del espacio conlleva la alteración del ojo fijo. Si cada interpretación es una perspectiva, no puede haber criterios externos y no hay forma de identificar que se trate del mismo objeto, ya no es el mismo ojo el que mira, por lo que ojo, espacio como organización y objeto son, aunque distantes, inseparables.

El ojo fijo no entra en el espacio, sólo lo hace como supuesto hipotético organizador del mismo. De ninguna manera puede ser conceptualizado, de lo contrario pertenecería a la reconstrucción y sería 
indistinguible de los objetos que hace posible; esto es, no sería ya ojo (sujeto), sino objeto; el ojo no puede mirarse a sí mismo. De esta manera, no podemos apelar a un sujeto para garantizar la identidad de objetos teóricos que pertenecen a diferentes espacios, pues, como dice Wittgenstein, el sujeto pensante, representante no existe (5.631). ${ }^{2}$ Cuando Moulines intenta interpretar -es decir, ofrecer un modelo operacionalel "constructor de mundo" de Carnap, el cual representa a un sujeto epistémico ideal, lo hace utilizando un sistema formal. Tal axiomatización generaría el ojo supuesto, pues consistiría en una elucidación formal del sujeto epistémico carnapiano (Moulines: 1996a). En tal caso, el constructor pierde su condición de sujeto-ojo para transformarse en objeto. Si coincidimos con Moulines en que "lo esencial de la "teoría de la constitución" del Aufbau es que ella presenta, por primera vez, un esbozo de formalización de lo que es un observador ideal" (1996a, p. 75), tal transformación del ojo en objeto del espacio de representación está presente en la obra mencionada de Carnap, dado que al proporcionar ésta las características esenciales del observador ideal estaría conceptualizando tal sujeto. De este modo, el sujeto epistémico se confunde entre los objetos que él mismo hace posible, se hace posible a sí mismo, es el ojo que se mira a sí mismo, entra en el sistema de representaciones.

Si yo escribiera un libro, "El mundo tal como lo encontré", en él habría que informar, entre otras cosas, sobre mi cuerpo y habría que decir cuáles son los miembros que están sujetos a mi voluntad y cuáles son los que no lo están, etc.; esto es, un método para aislar el sujeto o más bien para mostrar que, en un sentido relevante, no hay sujeto: de él solo, en efecto, no cabría tratar en este libro (Tractatus 5.631). El sujeto no pertenece al mundo, sino que es un límite del mundo (5.632). Dices que ocurre aquí enteramente como con el ojo y el campo visual, pero el ojo no lo ves realmente y nada en el campo visual permite inferir que es visto por un ojo" (5.633).

Si queremos evitar inconsistencias no se puede transformar ese modelo que Moulines construye para el sujeto constructor de mundo en el ojo de toda reconstrucción, pues ese modelo ya supone un esquema de representación.

La metateoría como una empresa hermenéutica significa que al interpretar formalizando se construye un espacio en donde la teoría in-

${ }^{2}$ Cfr. Moulines 1996, pp. 70-71. 
terpretada es un nuevo objeto teórico generado por ese espacio, por lo que no podemos justificar que reconstrucciones diferentes con aparatos formales diferentes sean del mismo objeto teórico. No hay un sujeto que nos garantice tal identidad, pues lo único que tenemos es este ojo inmóvil que con su mirada construye, pero que de ninguna forma podemos determinar ontológicamente, lo único que podemos decir de él son las condiciones formales que instituye en el espacio que hace posible la reconstrucción y eso es lo que se clarifica.

\section{Referencias}

Balzer, W., C.U. Moulines, y J. Sneed, 1987, An Architectonic for Science. The Structuralist Program, Reidel, Dordrecht.

Balzer, W. y P. Marcou, 1989, "A Reconstruction of Sigmund Freud's Early Theory of the Unconscious", en Westmeyer 1989, pp. 13-33.

Balzer, W., 1997, Teorías empíricas: modelos, estructuras y ejemplos, Alianza Editorial, Madrid.

Cirera, R., A. Ibarra y T. Mormann, 1996, El programa de Carnap. Ciencia, lenguaje y filosofía, Ediciones del Bronce, Barcelona.

Eliot, T.S., 1951, Poetry E Drama, Harvard University Press, Cambridge, Mass.

Goodman, N., 1968, Los lenguajes del arte. Aproximación a la teoría de los símbolos, Seix Barral, Barcelona.

—, 1978, Maneras de hacer mundos, Visor, Buenos Aires.

Gusdorf, G., 1988, Les origines de l'herméneutique, Payot, París.

Heisenberg, W., 1955, La imagen de la naturaleza en la física actual, Ediciones Orbis, Madrid.

Moulines, C.U., 1991, Pluralidad y recursión. Estudios epistemológicos, Alianza Editorial, Madrid.

—, 1996, "Las raíces epistemológicas del Aufbau de Carnap" en Cirera, Ibarra y Mormann 1996, pp. 45-74.

—, 1996, "Un modelo operacional del Aufbau de Carnap" en Cirera, Ibarra y Mormann 1996, pp. 75-90.

Panofsky, E., 1927, La perspectiva como forma simbólica, Tusquets, Barcelona.

Popkin, R., 2003, The History of Scepticism. From Savonarola to Bayle, Oxford University Press, Oxford.

Sneed, J., 1971, The Logical Structure of Mathematical Physics, Reidel, Dordrecht. 
Westmeyer, H. (comp.), 1989, Psychological Theories from a Structuralist Point of View, Springer-Verlag, Berlín.

Wittgenstein, L., 1997, Tractatus Logico-Philosophicus, Alianza Editorial, Madrid.

Recibido el 8 de septiembre de 2011

Aceptado el 30 de noviembre de 2011 